Cite this: Phys Chem Chem Phys, 2013, 15, 11006

Received 11th April 2013

Accepted 3rd May 2013

DOI: $10.1039 / \mathrm{c} 3 \mathrm{cp} 51542 \mathrm{~g}$

www.rsc.org/pccp

\section{Carrier dynamics in quantum-dot sensitized solar cells measured by transient grating and transient absorption methods $†$}

\author{
Naotaka Maeda, ${ }^{\text {ab }}$ Hiroaki Hata, ${ }^{\text {ab }}$ Naoya Osada, ${ }^{\text {ab }}$ Qing Shen, ${ }^{\text {bc }}$ Taro Toyoda, ${ }^{\text {bc }}$ \\ Shota Kuwahara ${ }^{a}$ and Kenji Katayama*a
}

Carrier dynamics in quantum-dot sensitized solar cells (QDSSCs) was clarified by combining the information obtained by the heterodyne transient grating (HD-TG), transient absorption (TA) and transient photocurrent (TP) measurements under the short circuit conditions in the time range from microseconds to seconds. The HD-TG signal is sensitive to the ionic species at the electrode/electrolyte interface, and the electrons in the titanium oxide layer injected from quantum dots (QDs) were monitored by the TA signal, and the photocurrent as a final output was monitored by the TP signal. By using the compensating information, the whole picture of the charge dynamics was obtained in the time region after the initial electron injection from QDs into the titanium oxide layer. In the former part of this paper, the assignment of the responses for each measurement was clarified based on the previous paper on dye sensitized solar cells (S. Kuwahara, et al. Phys. Chem. Chem. Phys., 2013, 15(16), 5975-5981). In the latter part, the effect of the device parameters for actual QDSSCs, such as electrolyte concentrations, and coating times of surface passivation of QDs were investigated.

\section{Introduction}

As promising devices for generating electricity from solar energy, dye-sensitized solar cells (DSSCs) have been intensively developed $^{1-6}$ and a number of them have been commercialized. In the last ten years, a new type of solar cells where quantum dots composed of inorganic materials (QDSSCs) were used as sensitizers, have been studied. Since QDs have some unique properties due to the quantum confinement effect, such as a tunable optical absorption spectrum, high absorption coefficient and high efficiency multiple exciton generation (MEG), ${ }^{7-9}$ the theoretical maximum efficiency for QDSSCs (44\%) is expected to be larger than that of DSSCs (30\%). ${ }^{10}$ However, QDSSCs present lower energy conversion efficiency at the moment. Typically InAs, CdS, CdSe, ${ }^{11,12}$ and $\mathrm{PbS}^{13}$ were used as QDs, but the efficiency for

\footnotetext{
${ }^{a}$ Department of Applied Chemistry, Chuo University, 1-13-27, Kasuga, Bunkyo, Tokyo 112-8551, Japan. E-mail: kkata@kc.chuo-u.ac.jp; Fax: +81-3-3817-1913; Tel: $+81-3-3817-1913$

${ }^{b}$ Department of Engineering Science, The University of Electro-Communications, 1-4-1 Chofugaoka, Chofu, Tokyo 182-8585, Japan.E-mail: shen@pc.uec.ac.jp ${ }^{c}$ CREST, Japan Science and Technology Agency (JST), 4-1-8 Honcho, Kawaguchi, Saitama 332-0012, Japan

† Electronic supplementary information (ESI) available. See DOI: 10.1039/ c3cp51542g
}

most QDSSCs was around 1-4\%. ${ }^{12,14,15}$ As for the other advantages of QDSSCs over DSSCs, the lower production cost and higher resistance to degradation are expected for QDSSCs because they are composed of inorganic materials.

To improve the conversion efficiency, many research studies have focused on the search for optimal conditions for QDs on the size,${ }^{16,17}$ binding on the substrate,${ }^{18}$ and for structure and materials of a semiconductor layer ${ }^{14,19-21}$ or their optimal combinations, ${ }^{11}$ but the understanding of the working mechanism is also necessary. The DSSCs and QDSSCs are very complicated electrochemical systems; typically a transparent electrode, a nanoporous semiconductor layer for electron transport such as $\mathrm{TiO}_{2}$, sensitizers, an electrolyte and redox solution, and a counter electrode. There are many electron or hole transfer processes involved in the photoelectrochemical process, and the time scale for these processes ranges from femtoseconds to seconds. ${ }^{22}$ Furthermore, not only an electron transfer in the expected direction, but also the back electron transfer or recombination processes are known to lower the cell efficiency. ${ }^{18,23,24}$

To investigate such processes of photogenerated carriers (electrons and holes), time-resolved measurement methods such as transient absorption (TA), ${ }^{2-24}$ transient photocurrent and transient photovoltage measurements have been generally used, ${ }^{25-28}$ and provided much information on the carrier 
dynamics of DSSCs and QDSSCs. However, each time-resolved method has some limitations to study the dynamics; for the TA method, optical absorption of the target molecules must be within the detection spectral range; intermediate states are not observed by current or voltage because they are the final output of a photovoltaic cell; and then it is difficult to clarify the whole process of carrier dynamics. As similar techniques in the frequency domain, intensity-modulated photovoltage and photocurrent spectroscopies, ${ }^{29,30}$ and impedance spectroscopy ${ }^{31}$ also have given fruitful information on the interfacial charge transfer, but they also do not give the whole information.

Although there are a number of studies of the carrier dynamics in DSSCs, there are much less data available for QDSSCs, and only a few groups lead this field. Hodes ${ }^{32}$ reviewed the difference of the carrier dynamics between DSSCs and QDSSCs, and it is explained that the main differences are caused by 1: the trap states at the $\mathrm{QD} /$ semiconductor interface, 2: different applicable electrolytes, and 3: multiple layers of QDs. The initial electron injection occurs on the picosecond order, where an electron-hole recombination process competes. There is another recombination process, where electrons injected into the semiconductor layer back to QDs via surface states. The holes generated in QDs are likely to be trapped on the picosecond time order, and they are transferred to redox species, whose charge transfer time ranges from picoseconds to milliseconds, depending on the redox species. ${ }^{33}$ Similarly to DSSCs, there is back electron transfer from the semiconductor to the electrolyte, competing with the electron diffusion in the semiconductor on the order of milliseconds. Different from DSSCs, the charge transfer time for each process has a large variation for QDSSCs probably due to the various QD states such as size, aggregation, etc. Such dynamic studies for QDSSCs are not so many, compared with the enormous number of studies for DSSCs. There are several important reports on carrier dynamics. Kamat, et $a l^{7,17,34}$ studied the initial electron injection dynamics depending on the size of QDs, and clarified that the electron injection rate was beautifully explained by the energy difference in the conduction band between QDs and semiconductors. Shen and Gomez et al. ${ }^{27,28,35}$ measured electron and hole dynamics, and clarified that holes are trapped by surface states of QDs and/ or scavenged by electrolytes within several picoseconds, and electrons are trapped at the interface and then injected into the semiconductor over several hundreds of picoseconds besides some direct injection of electrons within a few picoseconds. Although there have been studies on the carrier dynamics in QDSSCs on the picosecond time order, few data are available on the microsecond order, when back electron transfer, electrolyte diffusion, etc should be involved.

In the previous study, ${ }^{36,37}$ the carrier dynamics of DSSCs was investigated using the heterodyne transient grating (HD-TG) method in addition to TA and transient photocurrent (TP) methods on a nanoseconds to seconds time scale. Since the change in the refractive index is monitored using the HD-TG technique, various carrier dynamics can be monitored even if optical absorption is negligible, such as carrier transfer between $\mathrm{TiO}_{2}$, dyes and electrolyte species, carrier transport in a $\mathrm{TiO}_{2}$ layer or an electrolyte solution, etc. This advantage is suitable for DSSCs or QDSSCs, whose component materials or intermediate species do not necessarily have optical absorption, and such optically silent carrier dynamics is expected to be clarified using the TA method. In fact we could successfully observe the carrier dynamics at the electrode/electrolyte interface, especially on the electrolyte side; rearrangement or diffusion motion of charged electrolyte species occurred when the injected electrons were trapped at the $\mathrm{TiO}_{2}$ interface and when the reverse electron transfer occurred, which were not detected using the TA method. ${ }^{22}$ By combination of HD-TG, TA and TP measurements, we can expect to understand the whole dynamics in the time region from nanoseconds to seconds.

Similarly, in this study, we studied the carrier dynamics in QDSSCs using HD-TG, TA and TP methods, and clarified the dynamics involved in the working processes of QDSSCs in the temporal range from nanoseconds to seconds, from the investigations on the dependence on the applied voltage, concentration of electrolyte or redox species and the coating times of surface passivation of QDs.

\section{Experimental method}

The principle and the setup of the HD-TG method were reported in detail in the previous papers, ${ }^{36,38,39}$ and in ESI. $\dagger$ In this study, the pump light source was an OPO (Surelite II - 10FP) output excited by using a Nd:YAG laser (Panther, Continuum, ElectroOptics Inc.), and the pulse light had a wavelength of 500 or $570 \mathrm{~nm}$, a pulse width of $5 \mathrm{~ns}$, and a repetition rate of $0.5 \mathrm{~Hz}$. The repetition rate was decided as it is slow enough to recover the original baseline for the HD-TG and TA measurements. The pump intensity was $0.65 \mathrm{~mJ}$ pulse $^{-1}$ unless otherwise specified. The probe light was a fiber coupled CW semiconductor laser with a wavelength of $658 \mathrm{~nm}$ for all the HD-TG $(10 \mathrm{~mW})$ and the TA measurements $(4 \mathrm{~mW})$. The pump and probe lights were incident from the FTO substrate side to avoid the absorption of the pump light by a polysulfide redox solution. Transient photocurrent (TP) was measured using a potentiostat (Nikko Keisoku, time resolution: $10 \mu \mathrm{s}$ ), and it was recorded on an oscilloscope at the same time as the detection of the HD-TG response. TA responses were measured at the same probe wavelength just by removing a glass plate of a transmission grating used for the HD-TG measurement.

The method for the preparation of the working electrodes was reported in a previous paper. ${ }^{40}$ Aanatase $\mathrm{TiO}_{2}$ paste was prepared with $\mathrm{TiO}_{2}$ nanoparticles with an average diameter of $15 \mathrm{~nm}$ and polyethylene glycol with a molecular weight of 500000 . The resulting paste was cast onto a glass substrate coated with fluorine doped tin oxide (FTO) with a Scotch tape as a frame and spacer, raking off the excess solution with a glass rod (squeegee technique). The $\mathrm{TiO}_{2}$ electrodes were dried in air at room temperature for $10 \mathrm{~min}$, and annealed at $450{ }^{\circ} \mathrm{C}$ for $30 \mathrm{~min}$ in a furnace, and then cooled down to room temperature. The film thickness was around $7 \pm 1 \mu \mathrm{m}$. In the next step, CdSe was deposited on the electrode. The CdSe QDs were prepared using a chemical bath method, which was explained 
in the paper by Gorer and Hodes. ${ }^{41}$ Firstly, as the Se source, an $27 \mathrm{mM}$ sodium selenosulphate $\left(\mathrm{Na}_{2} \mathrm{SeSO}_{3}\right)$ solution was prepared by dissolving elemental Se powder in a $67 \mathrm{mM} \mathrm{Na} \mathrm{SO}_{3}$ solution. Secondly, $27 \mathrm{mM} \mathrm{CdSO}_{4}$ and $40 \mathrm{mM}$ trisodium salt of nitrilotriacetic acid $\left(\mathrm{N}\left(\mathrm{CH}_{2} \mathrm{CooNa}\right)_{3}\right)$ were mixed in a volume ratio of $1: 1$. Finally, both solutions were mixed in a volume ratio of $1: 2$. The $\mathrm{TiO}_{2}$ electrodes were placed in darkness in a glass container filled with the final solution for $24 \mathrm{~h}$. The samples were coated with $\mathrm{ZnS}$ for surface passivation of the QDs by using successive ionic layer adsorption and reaction

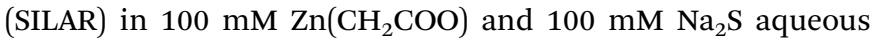
solution for $1 \mathrm{~min}$ for each dip. ${ }^{27}$ The coating procedure was typically repeated twice unless otherwise specified. We confirmed the surface topography using SEM measurements before and after the growth of CdSe quantum dots (Fig. S2 in ESI $\dagger$ ). The pore size of the electrode was on the order of tens of nanometers. The chemical composition of quantum dots was confirmed by XRD.

The electrolyte solution was a polysulfide aqueous solution ( $1 \mathrm{M}$ ) consisting of $1 \mathrm{M} \mathrm{Na}_{2} \mathrm{~S}$ and $1 \mathrm{M} \mathrm{S}$, unless otherwise specified. The sulfur ion species worked as both redox and electrolyte species. The counter electrode was copper sulfide $\left(\mathrm{Cu}_{2} \mathrm{~S}\right)$. The working electrode and the counter electrode were connected with a copper wire to a potentiostat, which was used for an ammeter, and no bias voltage was applied unless otherwise specified. The counter electrode was used as a reference electrode, too.

\section{Results and discussion}

\section{Assignment of the HD-TG, TA and TP responses}

Firstly, we investigated optimal pump irradiation intensity for dynamics measurements. The pump intensity dependencies of the HD-TG, TA and TP measurements were studied for the pump intensity range of $0.35-0.85 \mathrm{~mJ}$ pulse $\mathrm{e}^{-1}$ as shown in Fig. 1 . Basically the signal intensities for all the responses for the HD-TG, TA and TP measurements increased as the pump intensity, and the responses seemed to be saturated at higher intensities.

In all the measurements, the response waveforms showed discontinuous changes between 0.35 and $0.5 \mathrm{~mJ}$ pulse $^{-1}$. In the HD-TG responses, all the components in the response increased similarly; in the TA responses, a new component arose on the order of $10 \mathrm{~ms}$; TP increased suddenly and looked saturated for a pump intensity of over $0.5 \mathrm{~mJ}$ pulse $\mathrm{e}^{-1}$. It is supposed that there would be some threshold pump intensity for this QDSSC system.

As we explain later, the lifetime of electrons under this short circuit condition is on the order of several tens of milliseconds. When a single optical pulse with an intensity of $1 \mathrm{~mJ} \mathrm{pulse} \mathrm{p}^{-1}$ is irradiated during the lifetime, the corresponding irradiated photon number is roughly on the same order as that for 1 sun. (When an optical pulse with $1 \mathrm{~mJ}$ pulse $^{-1}$ is irradiated over an area of $1 \mathrm{~cm}^{2}$, the photon energy is equal to $10 \mathrm{~J} \mathrm{~m}^{-2}$, while 1 sun is equal to $1000 \mathrm{~W} \mathrm{~m}^{-2}$ and the irradiated photon number during $10 \mathrm{~ms}$ is $10 \mathrm{~J} \mathrm{~m}^{-2}$.) Considering the saturation of the photocurrent for the pump intensity $>0.5 \mathrm{~mJ}$ per pulse, the number of the injected electrons was enough to saturate the number of trapped electrons in $\mathrm{TiO}_{2}$, which build up the photovoltage to flow current. This condition is considered to
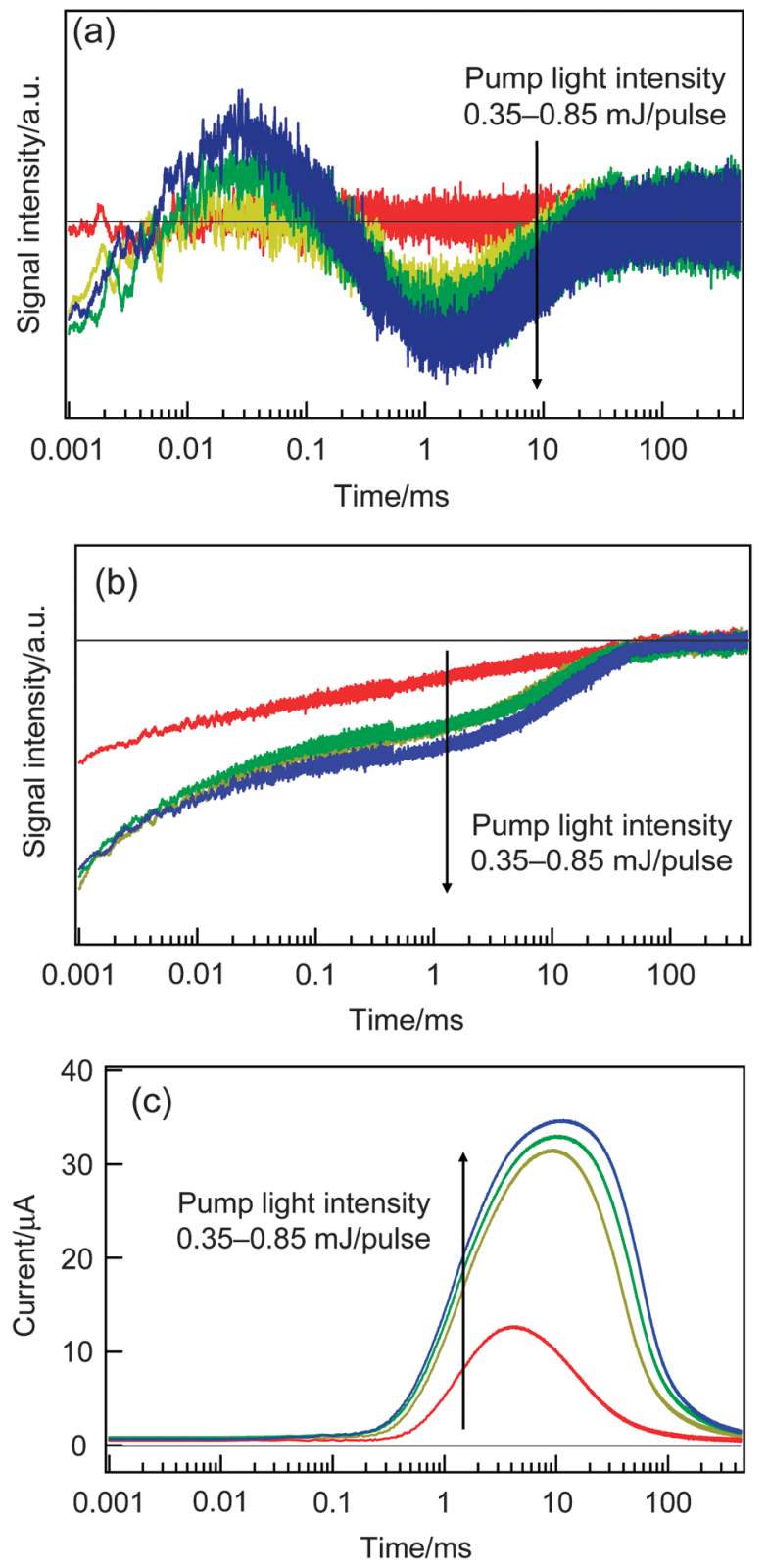

Fig. 1 The dependencies of the HD-TG (a), TA (b) and TP (c) responses on the pump intensities in the range from 0.35 to $0.85 \mathrm{~mJ}$ pulse $\mathrm{e}^{-1}$.

correspond to a typical working condition of this QDSSC. Since the temporal responses were the same for different intensities of the pump intensities $>0.5 \mathrm{~mJ} \mathrm{pulse} \mathrm{p}^{-1}$, the following experiments were performed for $0.65 \mathrm{~mJ}$ pulse $^{-1}$ throughout the experiments.

Next, the dependence on the materials of the counter electrodes was studied to check the effect of the reaction rate of redox species in solutions. At the counter electrodes, oxidized redox species at the working electrode are reduced to an original species, and if the reaction rate is not fast enough, the oxidized redox species are accumulated in the electrolyte solution and it may affect the HD-TG, TA and TP responses in the slower time region for repeated experiments. The influence was observed in the TP responses for a Pt counter 
electrode in polysulfide solution by repeating the pulse irradiation, as shown in Fig. S3 (ESI $\dagger$ ). By repeating the pulse irradiation, the photocurrent was gradually reduced. It is known that a $\mathrm{Cu}_{2} \mathrm{~S}$ counter electrode is more active for the redox reaction of polysulfide, compared with the Pt counter electrode, ${ }^{42}$ and the counter electrode was replaced with a $\mathrm{Cu}_{2} \mathrm{~S}$ counter electrode, which avoided the photocurrent reduction. On the other hand, the dependence on the counter electrode was not observed for the HD-TG responses for $\mathrm{Pt}$ and $\mathrm{Cu}_{2} \mathrm{~S}$ counter electrodes as shown in Fig. S4 (ESI $\dagger$ ). Even after 100 measurements, the difference was smaller than the error range and the effect of the counter electrode was negligible for the HD-TG responses.

The origin of the TA components was considered, and the components after microseconds were not caused by the excited holes left in QDs by initial electron injection into $\mathrm{TiO}_{2}$, which occurs within several picoseconds, ${ }^{27,28}$ and also were not caused by the excited state of QDs, which decays on the order of nanoseconds. ${ }^{7}$ The TA component in 1-100 ms was investigated. This component was observed for DSSCs in the previous paper, ${ }^{36}$ and was assigned to the decay of the trapped electrons in $\mathrm{TiO}_{2}$. To confirm the assignment, the applied voltage dependence was investigated. The dependencies of anodic and cathodic applied potentials are shown in Fig. 2(a) and (b), respectively. (It is noted that the solar cell typically works under cathodic applied potential in the range from $0-V_{\text {oc. }}$ ) When the absolute value of the applied potentials, $|V|$, was less than $0.5 \mathrm{~V}$,
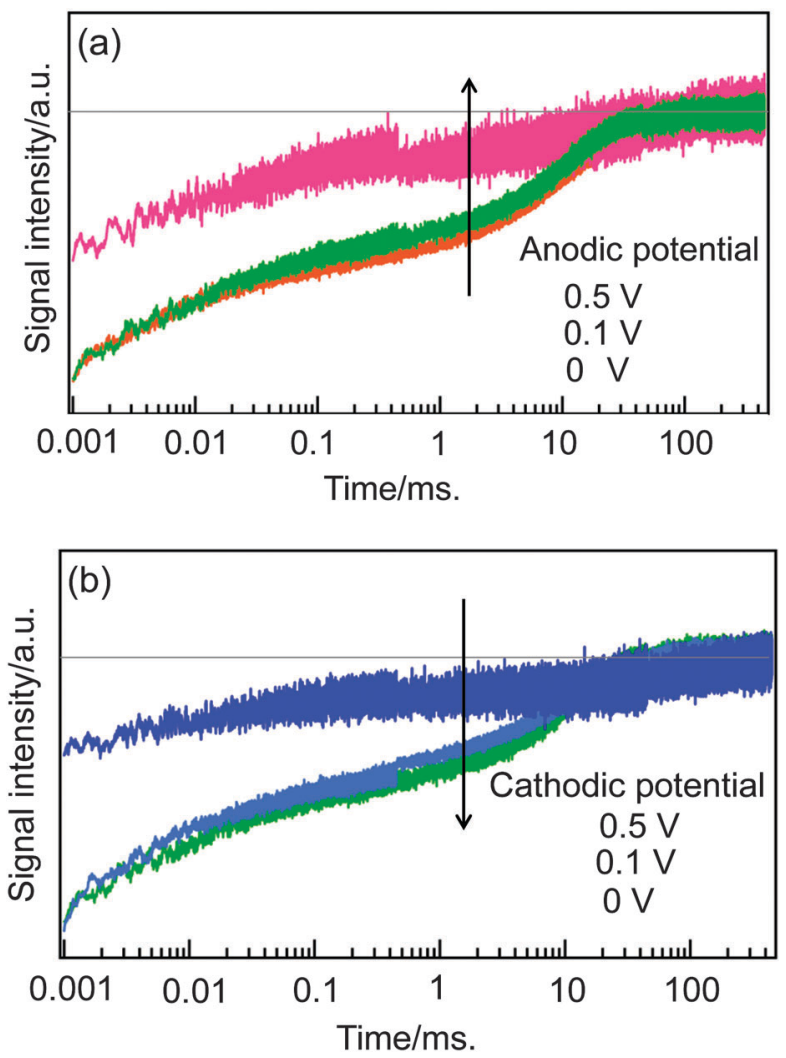

Fig. 2 Dependence of TA responses on the applied bias voltage is shown for anodic (a) and cathodic (b) applied potentials, respectively. the component in 1-100 ms was observed, but the component was lost for $|V|>0.5 \mathrm{~V}$. For the anodic applied potential, by decreasing the conduction band of $\mathrm{TiO}_{2}$, the trapped electrons were taken away from the circuit via the FTO electrode. For the cathodic applied potential, by increasing the conduction band of $\mathrm{TiO}_{2}$, the trapped electrons were taken by the redox species in the electrolyte solution. A weak response observed in the wide time range even for $|V|>0.5 \mathrm{~V}$ was assumed due to inhomogeneously distributed free electrons in $\mathrm{TiO}_{2}$. This cell structure has a $V_{\text {oc }}$ value of $0.52 \mathrm{~V}$, corresponding to the cathodic potential, and the electrons should be still trapped for a cathodic potential of $0.5 \mathrm{~V}$, but the electrons are taken away probably due to the non-perfect cell system designed for the HD-TG and TA measurements.

Next, the responses of the HD-TG and TA were compared in the presence and the absence of the working electrode, namely only the electrolyte solution and the same solution including the working electrode. In the absence of the working electrode, the pump wavelength was set at $500 \mathrm{~nm}$, and it was set at $570 \mathrm{~nm}$ in the presence of the working electrode. Since the absorbance of an electrolyte solution was 3.0 for $500 \mathrm{~nm}$, and 0.01 for $570 \mathrm{~nm}$, respectively, only the polysulfide was excited in the absence of the working electrode, and only the QDs were excited in the presence of the working electrode (the IPCE of this type of cell is shown in Fig. S5 in ESI $\dagger$ ).

By comparing the TA responses in the presence or absence of the electrode (Fig. 3), the first component (i) in 1-100 $\mu$ s was observed for both the cases, and the second component (ii) in 1-100 ms was observed only in the presence of the electrode. It is supposed that the (i) component corresponds to the decay of $\mathrm{S}^{-}$, which is generated via the oxidization of $\mathrm{S}^{2-}$ in the earlier time scale, because this component originates from the species in the electrolyte solution. ${ }^{35,43}$ In the case of the direct excitation of $\mathrm{S}^{2-}$, there are no reports on the formation of $\mathrm{S}^{-\bullet}$, but the similarity of the (i) component in both cases indicated that (i) has the same origin, $\mathrm{S}^{-\bullet}$. With regard to the (ii) component, as we have already explained, it is due to the trapped electrons in $\mathrm{TiO}_{2}$, and the fact that this component was observed only in the presence of the electrode supports the assignment.

By comparing the HD-TG responses in the presence or absence of the electrode, the first and third components, (I) and (III) were observed only in the presence of the electrode, and the second component (II) was observed in both cases. The (I) component had a time constant different from that of the (i) component in TA, and corresponds to the process after the electron injection from QDs and the following hole compensation from the electrolyte species, and also this occurred before the photocurrent increase in the circuit shown in Fig. 1(c). We assume that this process corresponds to the reorganization of electrolyte species at the electrode/electrolyte interface in order to compensate for the accumulated electron charge at the $\mathrm{TiO}_{2}$ surface. This is supported by the fact that similar dynamics was observed for DSSCs in the similar time region, reported recently. ${ }^{36}$ The (II) component corresponds to the thermal diffusion process in the electrode-electrolyte sample or the electrolyte solution, which is a typical response observed in the HD-TG signals, 

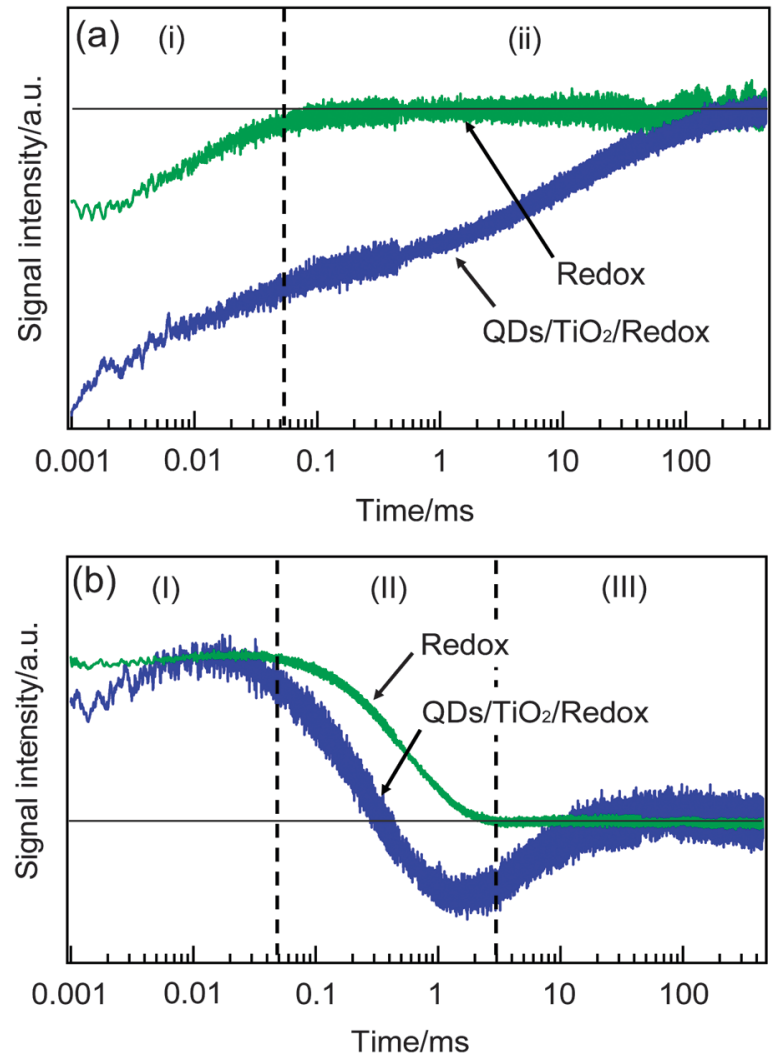

Fig. 3 The TA (a) and HD-TG (b) responses only for the redox solution and the QDSSC system including the working electrode. Time region was separated for each observed component for easy comparison.

originating from the refractive index change caused by the temperature change. The (III) component is the process only observed in the presence of the electrode, and the assignment was performed based on the following data.

To confirm the assignment, we compared the responses of HD-TG and TA when the electrolyte solution included electrolytes or not, as shown in Fig. 4. In the HD-TG responses, (I) and (III) components were observed only when the solution included electrolytes, while the (II) component was observed for both the cases. Since the (II) component was assigned to thermal diffusion processes in the electrode/solution sample, it is reasonable that the process was observed for both the cases. The electrolytes are necessary to observe (I) and (III) components, and it is reasonable that the (I) component corresponds to the reorganization process of redox species at the electrode/electrolyte interface. With regard to the (III) component, considering the time region on the order of 1-100 ms, which was on the same order as the photocurrent decay in the TP response and the decay of the trapped electrons of $\mathrm{TiO}_{2}$ observed in the TA response, it is related to the electron decay processes involving both the $\mathrm{TiO}_{2}$ layer and the redox species, thus it should be reverse electron transfer from $\mathrm{TiO}_{2}$ to redox species. The signal intensities for (I) and (III) were decreased when the solution was water, which can be understood that the trapped electrons in $\mathrm{TiO}_{2}$ were reduced due to the lack in counter cations and that caused to reduce the process of the reorganization of electrolyte species and the reverse electron transfer processes.
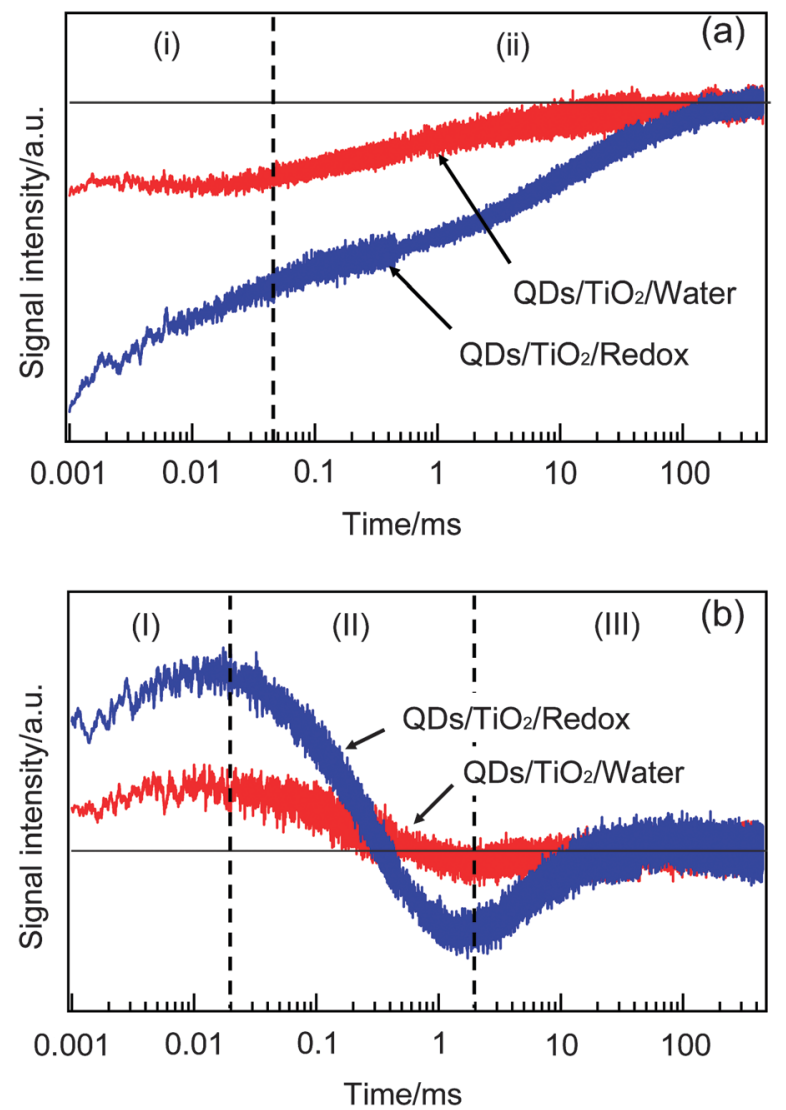

Fig. 4 The TA (a) and HD-TG (b) responses when the solution included electrolyte species or not are shown.

The signal intensity of (II) reflects the difference in the refractive index change due to thermal property of water and an electrolyte solution. ${ }^{44}$

As for the TA responses, the (i) component was lost and the (ii) component was reduced considerably in the absence of electrolyte species. Since the (i) component corresponds to the decay of $\mathrm{S}^{-*}$ species, the disappearance of the (i) component in the absence of redox species agrees with the assignment. The decrease in the signal intensity of (ii) is understood that a considerable number of electrons cannot be trapped stably in the absence of the counter cations.

\section{Dependence on various electrode and electrolyte conditions}

At first, we investigated how the electrolyte concentration affects the carrier dynamics. The HD-TG, TA, and TP results are shown in Fig. 5. In the HD-TG responses, with increasing electrolyte concentration the (I) process was accelerated; the signal intensity of (II) increased; the (III) signal intensity decreased and accelerated. The change in the (I) process indicated that the reorganization of electrolyte species at the interface became faster, and it is reasonable because the concentration of ionic species was increased. The increase in (II) intensity is just due to the optical property change due to the electrolyte concentration variation; the refractive index with respect to the temperature is typically positive for various electrolyte solutions, ${ }^{44}$ although there are no data available for 

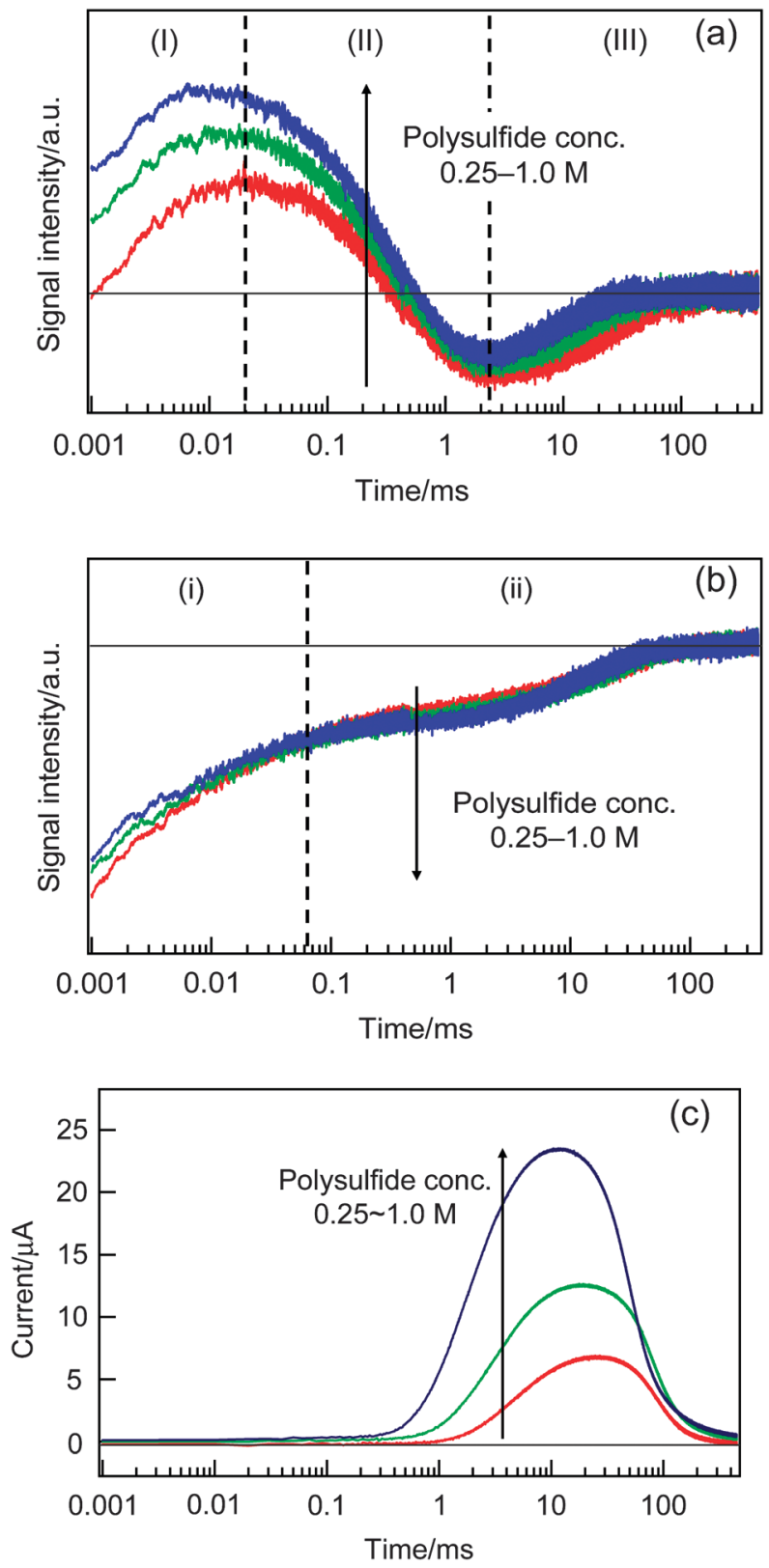

Fig. 5 The dependencies of the HD-TG (a), TA (b) and TP (c) responses on the polysulfide concentration.

polysulfide solutions. As for the (III) component, the acceleration of the process can be explained in the same manner as for the (I) component. The signal intensity decrease indicated the reduction of the electron acceptors (oxidized species), which are generated via the hole transfer from QDs to the redox species. It is supposed that the oxidized species at the interface disappeared more quickly by hole transfer due to higher redox concentration.

Looking at the TA responses, the (i) component decreased, while the (ii) component increased slightly as the concentration increased. Since the (i) component corresponds to the decay of $\mathrm{S}^{-}$, the signal intensity increase by increasing the electrolyte concentration is reasonable. The increase in the (ii) intensity indicated that the trapped electrons in $\mathrm{TiO}_{2}$ increased, which agreed with the HD-TG result that indicated that the reverse electron transfer processes were reduced, because more electrons can be retained in $\mathrm{TiO}_{2}$. The photocurrent increased and the rising time was accelerated with the concentration as shown in Fig. 5(c), indicating smoother hole acceptance by the redox species and faster ambipolar diffusion of electrons in the $\mathrm{TiO}_{2}$ layer as the electrolyte concentration increases.

Next, the effect of the ratio between $\mathrm{Na}_{2} \mathrm{~S}$ and $\mathrm{S}$ as an electrolyte solution was investigated (Fig. 6). The ratio was $1: 0,1: 0.25,1: 0.5$ and $1: 1$. By increasing the ratio of $\mathrm{S}$, a multi-atomic state, $\mathrm{Sn}^{2-}$ was increased and $\mathrm{S}^{2-}$ was decreased, and the former and the latter species work as an oxidized and reduced redox species, while the cation concentration was maintained to be $1 \mathrm{M}$. As the ratio of sulfur was increased, only the signal intensity of (II) was increased and the other components did not show any dependence on the HD-TG responses, and no dependence for all the components was observed on the TA responses, and a gradual increase in photocurrent was observed for the TP responses. The only change observed in the HD-TG response is probably due to the similar reason for the electrolyte concentration dependence; namely the temperature coefficient of the refractive index was different depending on the ingredients in the electrolyte solution, though there are no literature data to explain it. The photocurrent increase indicated the efficient hole acceptance by increasing the sulfur ratio. It was concluded that this ratio did not affect the carrier dynamics.

Finally, the effect of zinc sulfur coating was investigated. This coating is necessary for this kind of QDSSCs not only for preventing the corrosion of CdSe QDs, but also for stopping the reverse electron transfer from $\mathrm{TiO}_{2}$ to electrolytes, ${ }^{18}$ and actually the efficiency was doubled by this coating. Based on the SILAR method, ${ }^{45,46}$ the electrode was coated by ZnS on the order of a few nanometers in thickness, but the deposition times of $\mathrm{ZnS}$ is empirically decided and it is explained that the coating works for the blockage of the $\mathrm{TiO}_{2}$ surface and passivation of QDs, and also it promotes electron transfer. ${ }^{47}$ But the effect on the carrier dynamics is still not clear. We compared the HD-TG, TA and TP responses for different coating times, as shown in Fig. 7. By increasing the coating times, the signal intensity was gradually decreased, the (I) component was delayed and the intensity of the (III) component was much reduced in the HD-TG responses; the TA signal intensity was reduced, and the TP signal was reduced over 3 times coating.

The delay of the (I) component of the HD-TG signal can be understood that the coating of ZnS screened the charge of the trapped electrons in $\mathrm{TiO}_{2}$ from the electrolyte solution and that the effect of the Coulomb charge was weakened to de-accelerate the reorganization process of electrolyte species at the interface. The reduction of the (III) component indicated that the reverse electron transfer was blocked by the ZnS layer. The decrease in the TA and HD-TG signal intensity indicated that the trapped electrons were reduced by ZnS coating, and it is understood that the counter cations for stabilizing the trapped electrons in $\mathrm{TiO}_{2}$ cannot migrate into the $\mathrm{TiO}_{2}$ layer and a 

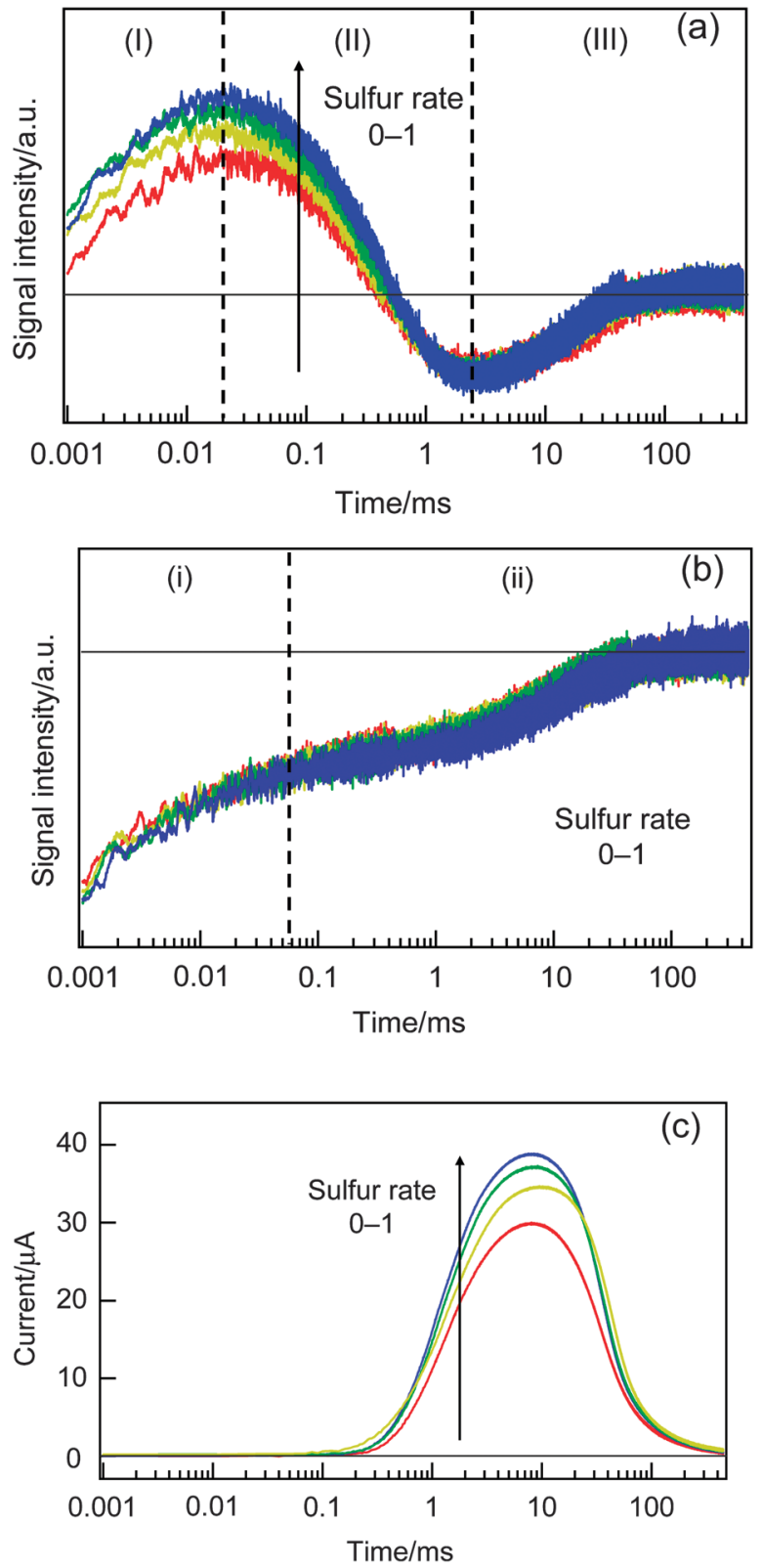

Fig. 6 The dependencies of the HD-TG (a), TA (b) and TP (c) responses on the ratio between $\mathrm{Na}_{2} \mathrm{~S}$ and $\mathrm{S}$ as an electrolyte solution.

smaller portion of electrons can stay in the $\mathrm{TiO}_{2}$ layer. The photocurrent was reduced due to the difficulty in charge transfer between the QDs and the redox species. Thus, it is concluded that the ZnS coating has a favorable effect on the reverse electron transfer, but has an unfavorable effect on the stabilization of trapped electrons and hole compensation of QDs, and the balance is important for optimization of the photovoltaic performance of QDSSCs.

\section{Conclusion}

We investigated the carrier dynamics in QDSSCs by combination of the HD-TG, TA, and TP methods. Since each
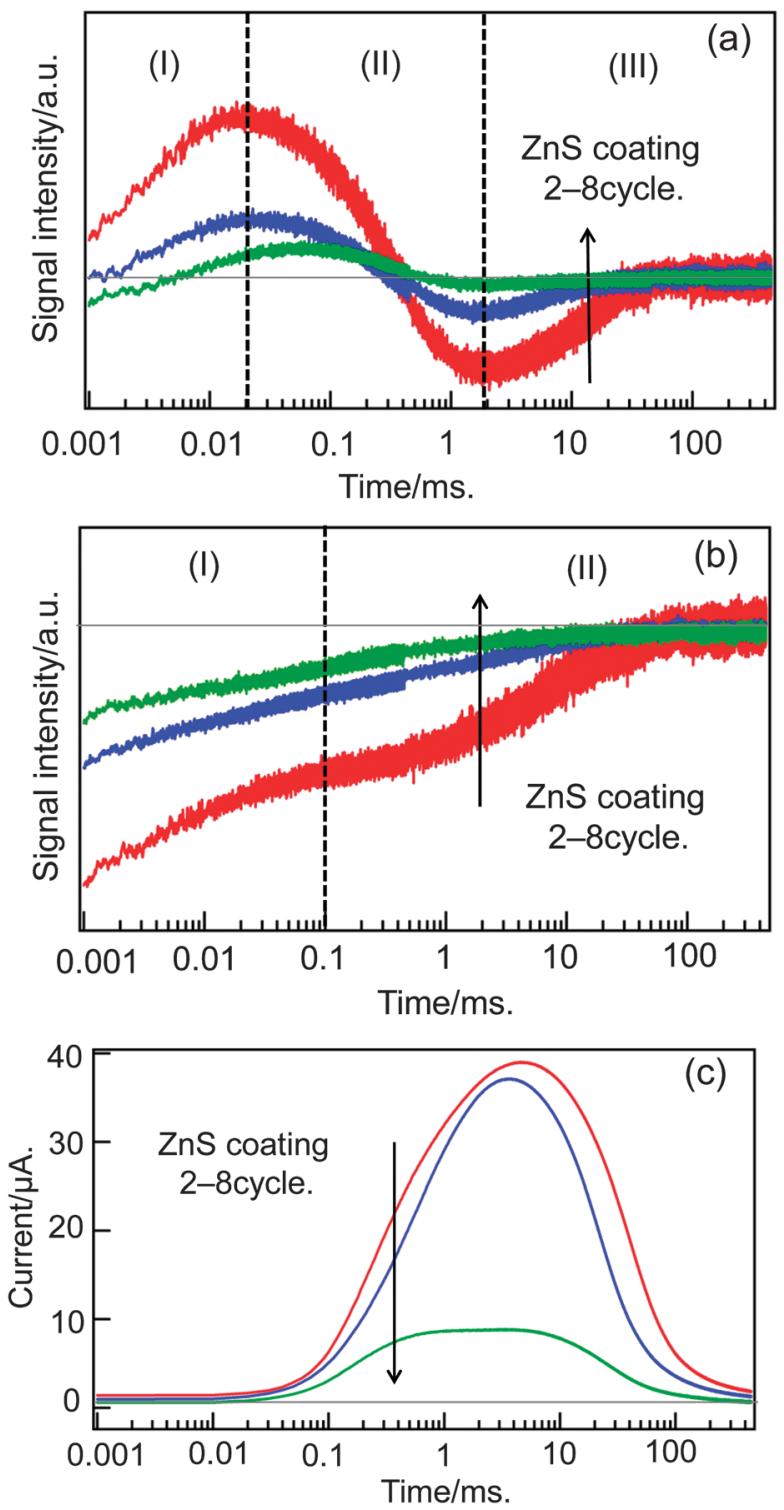

Fig. 7 The dependencies of the HD-TG (a), TA (b) and TP (c) responses on the times of ZnS coating.

technique gives different information, we could get the whole picture of the carrier dynamics in QDSSCs in the time range from microseconds to seconds. HD-TG gives information on the motion of ionic species at the interface; TA gives the electron lifetime in $\mathrm{TiO}_{2}$; $\mathrm{TP}$ gives the final output of current. It was clarified that this approach was effective not only for understanding the mechanism of the QDSSCs, but also for optimizing various device parameters. We expect that this approach will boost the development of new QDSSCs, and enhance their efficiency.

\section{Acknowledgements}

This research was supported by PRESTO and CREST programs of Japan Science and Technology Agency (JST). 


\section{Notes and references}

1 A. Hagfeldt, G. Boschloo, L. C. Sun, L. Kloo and H. Pettersson, Chem. Rev., 2010, 110, 6595.

2 T. W. Hamann, R. A. Jensen, A. B. F. Martinson, H. Van Ryswyk and J. T. Hupp, Energy Environ. Sci., 2008, 1, 66.

3 J. K. Lee and M. J. Yang, Mater. Sci. Eng., B, 2011, 176, 1142.

4 B. C. O'Regan and J. R. Durrant, Acc. Chem. Res., 2009, 42, 1799.

5 D. Wei, Int. J. Mol. Sci., 2010, 11, 1103.

6 Q. F. Zhang and G. Z. Cao, Nano Today, 2011, 6, 91.

7 P. V. Kamat, J. Phys. Chem. C, 2008, 112, 18737.

8 S. Ruhle, M. Shalom and A. Zaban, Chemphyschem, 2010, 11, 2290.

9 Q. Shen, K. Katayama, T. Sawada, S. Hachiya and T. Toyoda, Chem. Phys. Lett., 2012, 542, 89.

10 M. C. Hanna and A. J. Nozik, J. Appl. Phys., 2006, 100, 074510.

11 S. Gimenez, I. Mora-Sero, L. Macor, N. Guijarro, T. LanaVillarreal, R. Gomez, L. J. Diguna, Q. Shen, T. Toyoda and J. Bisquert, Nanotechnology, 2009, 20.

12 Q. X. Zhang, X. Z. Guo, X. M. Huang, S. Q. Huang, D. M. Li, Y. H. Luo, Q. Shen, T. Toyoda and Q. B. Meng, Phys. Chem. Chem. Phys., 2011, 13, 4659.

13 S. Hachiya, Q. Shen and T. Toyoda, J. Appl. Phys., 2012, 111. 14 S. Q. Huang, Q. X. Zhang, X. M. Huang, X. Z. Guo, M. H. Deng, D. M. Li, Y. H. Luo, Q. Shen, T. Toyoda and Q. B. Meng, Nanotechnology, 2010, 21.

15 Q. Shen, A. Yamada, S. Tamura and T. Toyoda, Appl. Phys. Lett., 2010, 97.

16 Q. Shen, J. Kobayashi, L. J. Diguna and T. Toyoda, J. Appl. Phys., 2008, 103.

17 I. Robel, M. Kuno and P. V. Kamat, J. Am. Chem. Soc., 2007, 129, 4136.

18 I. Mora-Sero, S. Gimenez, F. Fabregat-Santiago, R. Gomez, Q. Shen, T. Toyoda and J. Bisquert, Acc. Chem. Res., 2009, 42, 1848.

19 L. J. Diguna, Q. Shen, J. Kobayashi and T. Toyoda, Appl. Phys. Lett., 2007, 91.

20 T. Toyoda and Q. Shen, J. Phys. Chem. Lett., 2012, 3, 1885.

21 M. Samadpour, S. Gimenez, P. P. Boix, Q. Shen, M. E. Calvo, N. Taghavinia, A. I. Zad, T. Toyoda, H. Miguez and I. MoraSero, Electrochim. Acta, 2012, 75, 139.

22 A. Y. Anderson, P. R. F. Barnes, J. R. Durrant and B. C. O'Regan, J. Phys. Chem. C, 2010, 114, 1953.

23 S. Ardo, Y. Sun, F. N. Castellano and G. J. Meyer, J. Phys. Chem. B, 2010, 114, 14596.
24 J. G. Rowley and G. J. Meyer, J. Phys. Chem. C, 2011, 115, 6156.

25 P. R. F. Barnes, A. Y. Anderson, J. R. Durrant and B. C. O'Regan, Phys. Chem. Chem. Phys., 2011, 13, 5798.

26 H. K. Dunn and L. M. Peter, J. Phys. Chem. C, 2009, 113, 4726.

27 N. Guijarro, T. Lana-Villarreal, Q. Shen, T. Toyoda and R. Gomez, J. Phys. Chem. C, 2010, 114, 21928.

28 N. Guijarro, Q. Shen, S. Gimenez, I. Mora-Sero, J. Bisquert, T. Lana-Villarreal, T. Toyoda and R. Gomez, J. Phys. Chem. C, 2010, 114, 22352.

29 G. Schlichthörl, S. Y. Huang, J. Sprague and A. J. Frank, J. Phys. Chem. B, 1997, 101, 8141.

30 L. Dloczik, O. Ileperuma, I. Lauermann, L. M. Peter, E. A. Ponomarev, G. Redmond, N. J. Shaw and I. Uhlendorf, J. Phys. Chem. B, 1997, 101, 10281.

31 J. van de Lagemaat, N. G. Park and A. J. Frank, J. Phys. Chem. B, 2000, 104, 2044.

32 G. Hodes, J. Phys. Chem. C, 2008, 112, 17778.

33 N. S. Lewis, Annu. Rev. Phys. Chem., 1991, 42, 543.

34 A. Kongkanand, K. Tvrdy, K. Takechi, M. Kuno and P. V. Kamat, J. Am. Chem. Soc., 2008, 130, 4007.

35 Q. Shen, Y. Ayuzawa, K. Katayama, T. Sawada and T. Toyoda, Appl. Phys. Lett., 2010, 97, 263113.

36 S. Kuwahara, H. Hata, S. Taya, N. Maeda, Q. Shen, T. Toyoda and K. Katayama, Phys. Chem. Chem. Phys., 2013, 15, 5975.

37 Q. Shen, Y. Ogomi, B. W. Park, T. Inoue, S. S. Pandey, A. Miyamoto, S. Fujita, K. Katayama, T. Toyoda and S. Hayase, Phys. Chem. Chem. Phys., 2012, 14, 4605.

38 K. Katayama, M. Yamaguchi and T. Sawada, Appl. Phys. Lett., 2003, 82, 2775.

39 M. Yamaguchi, K. Katayama and T. Sawada, Chem. Phys. Lett., 2003, 377, 589.

40 Q. Shen and T. Toyoda, Thin Solid Films, 2003, 438-439, 167.

41 S. Gorer and G. Hodes, J. Phys. Chem., 1994, 98, 5338.

42 G. Hodes, J. Manassen and D. Cahen, J. Electrochem. Soc., 1980, 127, 544.

43 L. Min and R. J. D. Miller, Appl. Phys. Lett., 1990, 56, 524.

44 K. Katayama, T. Sawada, I. Tsuyumoto and A. Harata, Bull. Chem. Soc. Jpn., 1999, 72, 2383.

45 A. Braga, S. Giménez, I. Concina, A. Vomiero and I. n. MoraSeró, J. Phys. Chem. Lett., 2011, 2, 454.

46 H. M. Pathan and C. D. Lokhande, Bull. Mater. Sci., 2004, 27, 85. 47 N. Guijarro, J. M. Campina, Q. Shen, T. Toyoda, T. Lana-Villarreal and R. Gomez, Phys. Chem. Chem. Phys., 2011, 13, 12024. 